Journal of Animal and Veterinary Advances 10 (19): 2541-2545, 2011

ISSN: $1680-5593$

(C) Medwell Journals, 2011

\title{
Cryotop Device Enhances Vitrification Outcome of Immature Bovine Oocytes
}

\author{
${ }^{1} \mathrm{H}$. Hajarian, ${ }^{1} \mathrm{H}$. Wahid, ${ }^{1} \mathrm{Y}$. Rosnina, ${ }^{2} \mathrm{M}$. Daliri, ${ }^{1} \mathrm{M}$. Dashtizad, ${ }^{1} \mathrm{~T}$. Mirzapour, \\ ${ }^{1}$ N. Yimer, ${ }^{1}$ M.M. Bukar, ${ }^{1}$ M.I. Iswadi and ${ }^{3} \mathrm{O}$. Abas Mazni \\ ${ }^{1}$ Department of Veterinary Clinical Studies, Faculty of Veterinary Medicine, \\ Universitiy Putra Malaysia, 43400 Serdang, Selangor DarulEhsan, Malaysia \\ ${ }^{2}$ Department of Animal Reproduction, \\ National Institute of Genetic Engineering and Biotechnology, 14965 Tehran, Iran \\ ${ }^{3}$ Agro-Biotechnology Institute (ABI), 43400 Serdang, Selangor DarulEhsan, Malaysia
}

\begin{abstract}
The aim of this study was to evaluate the effectiveness of different cryodevices (Open Pulled Straw (OPS), Electron Microscopy Grid (EMG) and cryotop for vitrification of immature bovine oocytes. Polar body, MII stage, survivability and subsequent developmental rates were compared. Only oocytes with 4-5 layers of cumulus cells were used. Oocytes were equilibrated in the first vitrification solution (VS1; HS+10\% DMSO $+10 \%$ Ethylene Glycol (EG)) for 30-45 sec and then in the second vitrification solution (VS2; 20\% DMSO+20\% EG+0.5 $\mathrm{M}$ Sucrose) for $25 \mathrm{sec}$. Within $30 \mathrm{sec}$ they were mounted on one of the cryodevices and directly plunged into Liquid Nitrogen $\left(\mathrm{LN}_{2}\right)$ for 10 days. Immature oocytes vitrified using cryotop represented higher rate of polar body extrusion and nuclear maturity $(\mathrm{p}<0.05)$. The highest survivability resulted from cryotop and EMG groups and no significant difference found between them. Vitrified oocytes in cryotop group had highest cleavage and blastocyst rates. All of the mean measured rates for vitrified/warmed immature oocytes were significantly lower than that of control group $(\mathrm{p}<0.05)$. In conclusion, results of this study showed the superiority of cryotop device for vitrification of immature bovine oocytes which resulted in higher viability and subsequent embryo development.
\end{abstract}

Key words: Vitrification, cryodevice, bovine, immature oocytes, eggs, electron

\section{INTRODUCTION}

In 1985, vitrification has emerged as another option for cryopreservation of cells and organs (Rall and Fahy, 1985) and now is one of the focused areas in animal (Hadi et al., 2010a-c, 2011) and human reproductive biotechnologies. Formation of ice crystals is totally prevented in vitrification procedures using two main strategies: exceedingly high cooling/warming rates and highly viscose and concentrated vitrification solution (Smorag and Gajda, 1994; Vajta and Kuwayama, 2006). Some strategies such as reduction of vitrification solution volume (minimum volume $\leq 1 \mu \mathrm{L}$ ) and direct contact with liquid nitrogen or slush liquid nitrogen help to obtain necessary high cooling/warming rates (Yavin and Arav, 2007). The minimum volume-direct contact approach has been employing for cryopreservation of extremely chill sensitive drosophila eggs by loading them on electron microscopy grids (Mazur et al., 1992). Later on, Kuwayama et al. (2005) invented the cryotop method with the same technology like electron microscopy grid. The cryotop technology allows loading of oocytes or embryos with minimum vitrification solution. Direct contact of minimized vitrification solution $(\sim 0.1 \mu \mathrm{L})$ to liquid nitrogen results in exceedingly high cooling rate $\left(>23,000^{\circ} \mathrm{C}\right)$. Thereafter, the danger zone temperatures $\left(15-5^{\circ} \mathrm{C}\right)$ which attributed in chilling injury are easily skipped. Furthermore, minimizing of vitrification solution around the sample and direct contact facilitate achieving of extremely high warming rates $\left(>42,000^{\circ} \mathrm{C}\right)$ which has been found that it is more important to be fast enough and prevent devitrification at the time of warming (Isachenko et al., 2005a, b). Cryotop have shown its superiority to OPS for vitrification of immature pig (Liu et al., 2008), human (Kuwayama et al., 2005) and matured bovine oocytes (Morato et al., 2008) but there is still a gap of information for vitrification of immature bovine oocytes using different cryodevices.

Other issues that may profoundly affect the vitrification results are the species and developmental stages of oocyte or embryos. The reason is that permeability and sensitivity of gametes and embryos are

Corresponding Author: Abd Wahid Haron, Department of Veterinary Clinical Studies, Faculty of Veterinary Medicine, Universitiy Putra Malaysia, 43400 Serdang, Selangor DarulEhsan, Malaysia 
different with relation to the species and developmental stages (Hochi et al., 1998; Men et al., 2002; Magnusson et al., 2008). Previous studies have shown that $\mathrm{MII}$ bovine oocytes have greater permeability to cryoprotectants and water than GV oocytes.

The aim of the present study is to compare the efficiency of three cryodevices namely Open Pulled Straw (OPS), Electron Microscopy Grid (EMG) and cryotop for vitrification of immature bovine oocytes.

\section{MATERIALS AND METHODS}

Reagents: Unless otherwise indicated, all chemicals were purchased from Sigma-Aldrich (St. Louis, MO, USA).

Oocyte collection: Ovaries were collected from local abattoirs and transported to the laboratory within $3 \mathrm{~h}$ at $34-36^{\circ} \mathrm{C}$ in Phosphate-Buffer Saline (PBS; P-4417) containing penicillin-streptomycin $(100,000 \mathrm{IU}$ of penicillin and $100 \mathrm{mg}$ of streptomycin per liter). Cumulus-Oocyte Complexes (COCs) were obtained by slicing method in tissue culture medium 199 (Medium199, 12340; Gibco) containing $25 \mathrm{mM}$ HEPES, Earl's salts, L-glutamine and $2 \mathrm{mg} \mathrm{mL}^{-1}$ sodium bicarbonate modified by the addition of $4 \mathrm{mg} \mathrm{mL} \mathrm{m}^{-1}$ Bovine Serum Albumin (BSA, fraction $\mathrm{V}, \mathrm{A}-3311)$ and Gentamycin $50 \mu \mathrm{g} \mathrm{mL}^{-1}$ (G-1264).

Vitrification and warming: Recovered oocytes were washed twice in holding solution (HS, Hepes-buffered TCM medium supplemented with $20 \%$ Fetal Calf Serum, FCS) and kept there for about $15 \mathrm{~min}$. Group of four COCs were incubated in the first vitrification solution (VS1; 10\% DMSO and 10\% EG in WS) for 35-40 sec and then transferred to the second vitrification solution (VS2;20\% DMSO, 20\% EG and $0.5 \mathrm{M}$ sucrose in WS) for a further $25 \mathrm{sec}$. Instantly, oocytes were loaded on cryodevices including OPS, EMG and Cryotop (Kitazato Supply Co, Fujinomiya, Japan) and submerged into Liquid Nitrogen $\left(\mathrm{LN}_{2}\right)$ for storage. The time of exposure from VS2 to $\mathrm{LN}_{2}$ was not longer than $30 \mathrm{sec}$. Vitrified samples weremaintained in $\mathrm{LN}_{2}$ forat least 10 days.

Immediately after removing of cryodevices from $\mathrm{LN}_{2}$, they were submerged in $3 \mathrm{~mL}$ pre-warmed $\left(39^{\circ} \mathrm{C}\right) \mathrm{HS}$ plus $0.25 \mathrm{M}$ sucrose (T1) and smoothly tried to remove oocytes from cryodevices. Immature oocytes were left in $\mathrm{T} 1$ for $5 \mathrm{~min}$ and then transferred to HS plus $0.15 \mathrm{M}$ sucrose solution for $5 \mathrm{~min}$. Finally, the immature oocytes were washed twice in HM for $5 \mathrm{~min}$ each and processed for in vitro maturation, fertilization and culture.

In Vitro Maturation (IVM): Immature oocytes were washed 2 times in medium 199 supplemented with $4 \mathrm{mg} \mathrm{mL}^{-1} \mathrm{BSA}$ and washed again in maturation solution containing Hepes-buffered medium 199 supplemented with $10 \%$ fetal calf serum, $0.2 \mathrm{mM}$ sodium pyruvate (P-5280), $50 \mu \mathrm{g} \mathrm{mL}^{-1}$ Gentamycin (G-1264), $1 \mu \mathrm{g} \mathrm{mL}{ }^{-1}$ oestradiol-17 $\beta$ (E-8875) and $10 \mu \mathrm{g} \mathrm{mL}^{-1}$ insulin (I-6634; (Dashtizad et al., 2010). Approximately 20-30 oocytes were incubated in $400 \mu \mathrm{L}$ of maturation solution under mineral oil in 4 well plates for $22-24 \mathrm{~h}$ at $38.5^{\circ} \mathrm{C}$ under $5 \% \mathrm{CO}_{2}$ atmosphere with high humidity. The vitrified BCB-test separated oocytes also underwent the same maturation process with control group after warming.

Nuclear maturation stage: For the purpose of maturation determination, $24 \mathrm{~h}$ after maturation, oocytes were denuded using $0.1 \%(\mathrm{w} / \mathrm{v})$ hyaluronidase (Type 1-S) in Hepes-buffered medium 199 by vortexing. The cumulusfree oocytes were fixed in aceto-ethanol mixture $(1: 3, \mathrm{v} / \mathrm{v})$ at $5^{\circ} \mathrm{C}$ for $24 \mathrm{~h}$. Once fixed, oocytes in groups of 5-10 were mounted on slides. Cover-slip with 4 spots of silicon glued at the corners was gently pressed down until it touched and secured the oocytes. Afterward, acetolacmoid stain was passed under the cover-slip and remained there for $5 \mathrm{~min}$ followed by staining, a decolorizing solution (aceto-glycerol) was passed through to remove the stain residuals. Stained oocytes were examined under stereomicroscope $(40 \mathrm{x})$ for nuclear stages determination.

In Vitro Fertilization (IVF): The fertilization method was previously described by Parrish et al. (1988) with some modifications. Briefly, matured oocytes were washed 3 times in tyrode's albumin lactate pyruvate buffered with HEPES (Talp-Hepes) and twice in fertilization medium. Viable spermatozoa were obtained by centrifugation of frozen-thawed spermatozoa on Bovipure (Nidacon International $\mathrm{AB}$, Gothenburg, Sweden) discontinuous density gradient $(2 \mathrm{~mL}$ of top-layer over $2 \mathrm{~mL}$ of bottom-layer) for $20 \mathrm{~min}$ at $300 \times \mathrm{g}$ at room temperature. Viable spermatozoa collected at the bottom of the tube were washed with $5 \mathrm{~mL}$ of sperm-TALP supplemented by $6 \mathrm{mg} \mathrm{mL}^{-1} \mathrm{BSA}$ (fraction V, A-3311) and centrifuged for $10 \mathrm{~min}$ at $300 \times \mathrm{g}$. After centrifugation $150 \mu \mathrm{L}$ of the pellet was selected.

Spermatozoa were counted in a hemacytometer and diluted in the appropriate volume of IVF-Talp to give a concentration of $1 \times 10^{6}$ spermatozoa $\mathrm{mL}^{-1}$. IVM-oocytes were transferred in groups of up to 7 into $50 \mu \mathrm{L}$ of fertilization solution supplemented with $10 \mu \mathrm{g} \mathrm{mL}^{-1}$ heparin-sodium salt (Sigma, H-3393). Spermatozoa suspension was added to each fertilization droplet. Oocytes and sperms were incubated for $18 \mathrm{~h}$ at $38.5^{\circ} \mathrm{C}$ in $5 \% \mathrm{CO}_{2}$ in humidified air. 
In Vitro Culture (IVC): About $18 \mathrm{~h}$ after IVF, oocytes were freed of cumulus cells by high speed vortexing and washed twice in Talp-Hepes and culture medium. For each well, 10-15 oocytes were transferred to $400 \mu \mathrm{L}$ of Synthetic Oviductal Fluid (SOFaaci) supplemented with 5\% adult bovine serum (B-9433) and $1 \mu \mathrm{g} \mathrm{mL}$ gentamycin under mineral oil. Every 2 days after trans fer to culture medium, $200 \mu \mathrm{L}$ of culture medium replenished with $200 \mu \mathrm{L}$ fresh incubated SOF medium. Cleavage and blastocyst rates were recorded at $48 \mathrm{~h}, 7$ and 9 days post insemination, respectively.

Experimental design: Two experiments were performed to find out the in vitro viability of immature oocytes after vitrification using EMG, cryotop and OPS. In the first experiment, some of the vitrified/warmed immature oocytes were matured and freed of cumulus cells at 22-24 h of maturation. Oocytes were monitored for the presence of polar body. Afterward, the oocytes were fixed and stained. Nuclear maturation stages for each group were determined under the light microscope. In the second experiment, survivability, cleavage $\geq 8$ cell and blastocyst rates were evaluated at $24 \mathrm{~h}$ after IVM, 40-48 h post insemination (hpi), 4-5 days post insemination (dpi) and 7-10 dpi, respectively.

Statistical analysis: All experiments were repeated 6 times. Significant differences among treatments were revealed by non parametric one-way analysis of variance followed by Duncan's Multiple Range test for mean comparisons $(\mathrm{p}<0.05)$ using SAS software ver. 9.1 (SAS Inst., Cary, NC).

\section{RESULTS AND DISCUSSION}

Polar body assessment of denuded oocytes revealed that vitrification using cryotop increased the percentage of oocytes with extruded polar body ( $<<0.05$; Table 1$)$. Polar body rate was significantly lower in cryotop when it compared with control group $(\mathrm{p}<0.05)$. Although, degeneration happened in higher rate in OPS group $(\mathrm{p}<0.05)$, control group showed the least degeneration followed by cryotop. There was no significant difference regarding the absence of Polar Body ( $\mathrm{PB}-$ ) between OPS, cryotop and EMG groups $(\mathrm{p}>0.05)$ but control group represented the lowest percentage of $\mathrm{PB}$ compared with other groups $(\mathrm{p}<0.05$; Table 1$)$.

Vitrified/warmed immature oocytes in Cryotop group were seen in MII stage of maturation (58\%) significantly higher compared with EMG and OPS groups (46\% and $35 \%$, respectively). Oocytes in Cryotop and EMG groups
Table 1: Polar body rate after in vitro maturation in different cryodevices Polar \begin{tabular}{lcccc} 
body rates & OPS $(\mathrm{n}=49)$ & Cryotop $(\mathrm{n}=58)$ & EMG $(\mathrm{n}=77)$ & Control $(\mathrm{n}=69)$ \\
\hline Polar body + & $10(26 \%)^{\mathrm{c}}$ & $24(41 \%)^{\mathrm{b}}$ & $24(32 \%)^{\mathrm{c}}$ & $48(70 \%)^{\mathrm{a}}$
\end{tabular} Polar body - $\quad 27(50 \%)^{\mathrm{a}} \quad 26(45 \%)^{\mathrm{a}} \quad 39(52 \%)^{\mathrm{a}} \quad 20(29 \%)^{\mathrm{b}}$

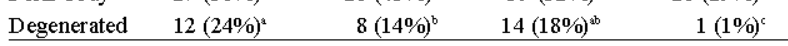
Data were pooled from 4 replicates; ".c Values with different superscripts in the same row are significantly different; ( $p<0.05$; ANOVA and Duncan's test)

Table 2: Nuclear maturation of vitrified immature oocytes

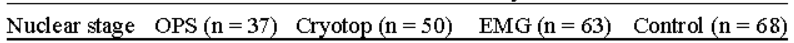
\begin{tabular}{lllll}
\hline $\mathrm{GV}$ & $15(41 \%)^{\mathrm{a}}$ & $8(16 \%)^{\mathrm{c}}$ & $16(25 \%)^{\mathrm{b}}$ & $4(6.0 \%)^{\mathrm{d}}$
\end{tabular}

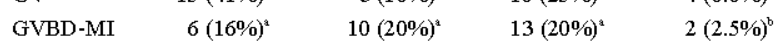
MII $\quad 13(35 \%)^{\mathrm{d}} \quad 29(58 \%)^{\mathrm{b}} \quad 29(46 \%)^{\mathrm{c}} \quad 60(88.0 \%)^{\mathrm{a}}$

\begin{tabular}{|c|c|}
\hline Unclassified & \\
\hline
\end{tabular}

Data were pooled from 4 replicates; ${ }^{a-d}$ Values with different superscripts in the same row are significantly different $(\mathrm{p}<0.05)$

tended to pursue their maturation and had less GV stage oocytes in comparison to OPS group ( $<<0.05$; Table 2$)$. There was no significant difference in percentage of GVBD-MI among all treatment groups. In comparison with the other groups, the least GV and GVBD-M rate resulted from control group $(\mathrm{p}<0.05)$.

Table 3 shown the results obtained from viability, cleavage $>8$ cells and blastocyst rates of vitrified/warmed immature bovine oocytes. It is apparent from this table that oocytes from cryotop and EMG group (90 and 89\%, respectively) had significantly higher viability compared with OPS group $(79 \% ; \mathrm{p}<0.05)$. However, viability rate of control group (100\%) was significantly higher than treated groups. Some of the treated oocytes lost their survivability during IVF. Presence of multiple spermatozoa in the perivitelline space of vitrified/warmed oocytes was also noticeable. The rate of cleaved oocytes were significantly greater in the cryotop group (40.21 $\pm 4.21 \%$ ) compared to EMG and OPS (33.29 \pm 1.35 and $30.94 \pm 1.61$, respectively) but there was no difference between $\mathrm{EMG}$ and OPS groups. Developmental rate of embryos to 8 cell or beyond was significantly greater $(\mathrm{p}<0.05)$ in Cryotop group $(23.33 \pm 1.62 \%)$ than other groups $(17.15 \pm 3.22 \%$, $12.91 \pm 2.00 \%$ for OPS and EMG, respectively). Although, no significant difference in blastocyst formation was revealed between treated groups $(\mathrm{p}<0.05)$, Cryotop resulted in higher percentage of blastocyst rate compared with other groups ( $>>0.05$; Table 3 ). All of the mean measured rates for vitrified/warmed immature oocytes were significantly lower than that of control group $(\mathrm{p}<0.05)$.

In this study, the researchers have compared three efficient cryodevices for vitrification of immature bovine oocytes. The reason was to standardize usage of a cryodevice which results in a higher cooling/warming rate, 
J. Anim. Vet. Adv., 10 (19): 2541-2545, 2011

\begin{tabular}{|c|c|c|c|c|c|}
\hline Treatments & No. of oocytes & Viability (\%) & Oocytes cleaved (\%) & $\geq 8$ cell embryos $(\%)$ & Blastocyst (\%) \\
\hline Control & 174 & $100^{a}$ & $75.70 \pm 3.93^{\mathrm{a}}$ & $61.46 \pm 3.10^{9}$ & $35.62 \pm 2.06^{\mathrm{a}}$ \\
\hline OPS & 133 & $79.63 \pm 2.06^{c}$ & $30.94 \pm 1.61^{c}$ & $12.91 \pm 2.00^{\mathrm{d}}$ & $2.85 \pm 2.27^{b}$ \\
\hline Cryotop & 104 & $90.34 \pm 2.49^{b}$ & $40.21 \pm 4.21^{b}$ & $23.33 \pm 1.62^{b}$ & $4.88 \pm 2.72^{b}$ \\
\hline EMG & 153 & $89.20 \pm 2.36^{b}$ & $33.29 \pm 1.35^{c}$ & $17.15 \pm 3.22^{c}$ & $3.61 \pm 2.16^{b}$ \\
\hline
\end{tabular}

Data were pooled from 6 replicates; ${ }^{a-d}$ Values with different superscripts in the same column are significantly different $(p<0.05)$

better protection and higher viability after warming. Cryotop resulted in higher viability compared to other treated groups. Survivability of immature bovine oocytes was assessed by recovery of oocytes after warming, existence of polar body and by staining for nuclear maturation. Nuclear maturation rate of vitrified immature oocytes by cryotop device was comparably higher than other cryodevices demonstrating the ability of immature oocytes to survive vitrification. Nuclear maturity of vitrified immature bovine oocytes were comparably lower in previous published studies (Cetin and Bastan, 2006; Yamada et al., 2007) than this experiment.

The two major obstacles which overcome by vitrification procedure are ice crystal formation and chilling injury (Vajta and Kuwayama, 2006). Chilling injury mostly affects cytoskeleton and cell membranes including oolema and membrane of organelles (Morato et al., 2008). For vitrification process and prevention of intracellular ice formation, two main approaches are necessary which are high concentrations of permeating cryoprotectants (CPAs, around 6-8 M) and high cooling/warming rates. The higher viability resulted from cryotop and EMG represent improvements over the rates obtained by OPS device and can be attributed to the higher cooling/warming rates obtained using the cryotop and EMG technology. Electron microscopy grid has been employed for cryopreservation of extremely chill sensitive drosophila eggs by loading them on EMG (Mazur et al., 1992). It has been said that the basis of cryotop device was obtained from EMG device. On the other hand, the Cryotop technology (Kuwayama et al., 2005) allows loading of oocytes or embryos with minimum vitrification solution. Even after mounting of sample on cryotop the excessive amount of vitrification solution is aspirated. Direct contact of minimized vitrification solution $(\sim 0.1 \mu \mathrm{L})$ to liquid nitrogen results in exceedingly high cooling rate $\left(>23,000^{\circ} \mathrm{C}\right)$. Thereafter, the danger zone temperatures $\left(15-(-5)^{\circ} \mathrm{C}\right)$ which attributed in chilling injury are easily skipped. Furthermore, minimizing of vitrification solution around the sample facilitates achieving of extremely high warming rates $\left(>42,000^{\circ} \mathrm{C}\right)$ which has been found that it is more important to be fast enough and prevent devitrification at the time of warming (Isachenko et al., 2005a, b).
The percentage of cleaved oocytes and $\geq 8$ cell embryos were higher in cryotop followed by EMG. Hence, it seems that cryotop could better preserve the cell structure and consequently fertilization capacity of vitrified immature oocytes. However, these results were significantly lower than those of fresh immature oocytes (control group). This reduction in cleavage rate of EMG group oocytes and subsequent development of them might be a result of mishandling of the device in which the EMG are tiny and have to be delicately handled. At the time of warming, some of them positioned face down and subsequently damage the oocytes. At warming time, EMGs should be handled using a fine forceps pre-equilibrated in liquid nitrogen. In some cases because of boiling of liquid nitrogen and searching for tiny grid and also transmission of body temperature to the forceps, the temperature of forceps goes up and at the time of grabbing the grid which creates a temperature shock and may cause devitrification. These results therefore need to be interpreted with caution.

Although, the percentage of blastocyst formation was not significantly different, Cryotop resulted in higher blastocyst rate and showed its superiority over the other two cryodevices. However, comparison of blastocyst formation between treated groups and fresh group indicated that damage resulted from vitrification could compromise the viability and subsequent development of the vitrified immature oocytes. These results are consistent with the results from Morato et al. (2008) and Chian et al. (2004) which reported the superiority of cryotop versus OPS.

\section{CONCLUSION}

In this study, the minimum volume-direct contact approach employed for cryotop technology may provide enough support for vitrification of immature bovine oocytes. Further studies to employ cryotop technology using its improved vitrification solution needs to be performed which may provide better environment for vitrification of immature bovine oocytes and its subsequent development.

\section{ACKNOWLEDGEMENTS}

This research was supported by a research grant from Research University Grant Scheme, Universiti Putra Malaysia (vote no. 9169500). 


\section{REFERENCES}

Cetin, Y. and A. Bastan, 2006. Cryopreservation of immature bovine oocytes by vitrification in straws. Anim. Reprod. Sci., 92: 29-36.

Chian, R.C., M. Kuwayama, L. Tan, J. Tan, O. Kato and T. Nagai, 2004. High survival rate of bovine oocytes matured in vitro following vitrification. $\mathrm{J}$. Reprod. Dev., 50: 685-696.

Dashtizad, M., A.W. Haron, R. Yusoff, M. Daliri and H. Hajarian et al., 2010. Synergistic effect of insulin on in vitro development of immature bovine oocytes. Am. J. Anim. Vet. Sci., 5: 258-265.

Hadi, H., H. Abd Wahid, Y. Rosnina, M. Daliri, M. Dashtizad, R. Holmes and O. Abas Mazni, 2011. Nuclear maturation of immature bovine oocytes after vitrification using open pulled straw and cryotop methods. Afr. J. Biotechnol., 10: 6-6.

Hadi, H., H. Wahid, O.A. Mazni, Y. Rosnina and M. Daliri et al., 2010a. Effect of equilibration temperature on in vitro viability and subsequent embryo development of vitrified-warmed immature bovine oocytes. Am. J. Anim. Vet. Sci., 5: 71-75.

Hadi, H., H. Wahid, O.A. Mazni, Y. Rosnina, M. Daliri and M. Dashtizad, 2010b. Effects of exposure to dmso in vitrification solution on cytotoxicity and in vitro viability of immature bovine oocytes. Reprod. Biomed. Online, 20: S37-S38.

Hadi, H., H. Wahid, Y. Rosnina, M. Daliri and M. Dashtizad et al., 2010c. Selection of immature bovine oocytes using brilliant cresyl blue enhances nuclear maturity after vitrification. J. Anim. Vet. Adv., 9: 2710-2713.

Hochi, S., K. Ito, M. Hirabayashi, M. Ueda, K. Kimura and A. Hanada, 1998. Effect of nuclear stages during IVM on the survival of vitrified-warmed bovine oocytes. Theriogenology, 49: 787-796.

Isachenko, V., M. Montag, E. Isachenko and H. van der Ven, 2005b. Vitrification of mouse pronuclear embryos after polar body biopsy without direct contact with liquid nitrogen. Fertil. Steril., 84: 1011-1016.

Isachenko, V., M. Montag, E. Isachenko, V. Zaeva, I. Krivokharchenko, R. Shafei and H. van der Ven, 2005a. Aseptic technology of vitrification of human pronuclear oocytes using open-pulled straws. Hum. Reprod., 20: 492-496.
Kuwayama, M., G. Vajta, O. Kato and S.P. Leibo, 2005. Highly efficient vitrification method for cryopreservation of human oocytes. Reprod. Biomed. Online, 11: 300-308.

Liu, Y., Y. Du, L. Lin, J. Li and P.M. Kragh et al., 2008. Comparison of efficiency of Open Pulled Straw (OPS) and Cryotop vitrification for cryopreservation of in vitro matured pig oocytes. Cryo. Lett., 29: 315-320.

Magnusson, V., W.B. Feitosa, M.D. Goissis, C. Yamada and L.M.T. Tavares et al., 2008. Bovine oocyte vitrification: Effect of ethylene glycol concentrations and meiotic stages. Anim. Reprod. Sci., 106: 265-273.

Mazur, P., K.W. Cole, J.W. Hall, P.D. Schreuders and A.P. Mahowald, 1992. Cryobiological preservation of Drosophila embryos. Science, 258: 1932-1935.

Men, H., R.L. Monson and J.J. Rutledge, 2002. Effect of meiotic stages and maturation protocols on bovine oocyte's resistance to cryopreservation. Theriogenology, 57: 1095-1103.

Morato, R., D. Izquierdo, M.T. Paramio and T. Mogas, 2008. Cryotops versus Open-Pulled Straws (OPS) as carriers for the cryopreservation of bovine oocytes: Effects on spindle and chromosome configuration and embryo development. Cryobiology, 57: 137-141.

Parrish, J.J., J. Susko-Parrish, M.A. Winer and N.L. First, 1988. Capacitation of bovine sperm by heparin. Biol. Reprod., 38: 1171-1180.

Rall, W.F. and G.M. Fahy, 1985. Ice-free cryopreservation of mouse embryos at -196 degrees $\mathrm{C}$ by vitrification. Nature, 313: 573-575.

Smorag, Z. and B. Gajda, 1994. Cryopreservation of mammalian ova and embryos by vitrification. Biotechnol. Adv., 12: 449-465.

Vajta, G. and M. Kuwayama, 2006. Improving cryopreservation system. Theriogenology, 65: $236-244$.

Yamada, C., H.V.A. Caetano, R. Simoes, A.C. Nicacio and W.B. Feitosa et al., 2007. Immature bovine oocyte cryopreservation: Comparison of different associations with ethylene glycol, glycerol and dimethylsulfoxide. Anim. Reprod. Sci., 99: 384-388.

Yavin, S. and A. Arav, 2007. Measurement of essential physical properties of vitrification solutions. Theriogenology, 67: 81-89. 